\title{
Nouvelles sources photoniques VUV à pompage collisionnel
}

\author{
B. Fontaine et M. Sentis
}

IMFM-UM34 CNRS, Pole Scientifique de Luminy, case 918, 13288 Marseille Cedex 09, France

\begin{abstract}
Recent progress achieved in the international community on pulsed collisionally excited coherent and incoherent primary sources emitting at very short wavelength are reviewed. Enphasis is made on $\mathrm{Rg}_{2}$ rare gas excimers, rare gas-alkali and alkali-halogen ionic excimers, UV-VUV emissions from surface discharge, and long pulse high efficiency $\mathrm{KrCl}$ excimer laser $(\lambda=222 \mathrm{~nm})$. These systems are discussed as well as their perspectives and potential applications.
\end{abstract}

\section{1.- Introduction}

Depuis une quinzaine d'années la communauté scientifique des physiciens s'intéresse activement aux processus physiques pouvant mener à des émissions cohérentes et incohérentes intenses dans le domaine spectral de l'ultraviolet lointain et si possibles accordables en longueur d'onde. Ces recherches sont fortement motivées par d'importantes applications potentielles de ces sources dans des domaines aussi variés que le traitement des matériaux, la photochimie, la biologie, etc ${ }^{\uparrow-5}$.

L'objet du présent article est de présenter les progrès récents obtenus, au plan international, sur de nouvelles sources photoniques prometteuses émettant dans le domaine spectral de l'ultraviolet lointain $(\lambda \simeq 50-200 \mathrm{~nm})$ à la suite de processus collisionnels induits dans des milieux gazeux à haute pression par une excitation au moyen de systèmes électriques ou optiques impulsionnels (faisceau d'électrons relativistes ${ }_{*}$ décharge, faisceaџ de rayons $X)$. Le cas des lasers à exciplexes ou assimilés UV-VUV $\mathrm{XeF}^{*}{ }^{*} \mathrm{XeCl}^{*}, \mathrm{KrF}^{*}, \mathrm{ArF}^{*}$, $\mathrm{F}^{*}{ }^{*}$ étant traité dans un autre article du même numéro du J. de Physique ${ }^{6}$, n'est pas abordé ici. Un effort particulier est fąit sur la description des systèmes photoniques VUV suivants: (a) excimères de gaz rare $\mathrm{Rg}_{2}$, (b) exciplexes ioniques gaz rare-alcalin $\mathrm{Rg}^{+} \mathrm{A}$, (c) exciplexes ioniques alcalin-halogène $A+X$, (d) sources photoniquęs issues d'une décharge sur une ferrite préalablement formée, (e) laser à exciplexes $\mathrm{KrCl}^{*}(\lambda=222 \mathrm{~nm})$ à rendement élevé en régime d'impulsions longues, (f) quelques autres sources VUV primaires.

\section{Excimères de gaz rares}

Après avoir donné de grands espoirs en tant que source cohérente accordable de grande puissance et rendement élevé dans le VUV au moment de leur découverte en 1973 (excitation par un faisceau d'électrons relativistes pulsé intense ${ }^{7,8}$, , les lasers à excimères de gaz rares ont montré rapidement leur limitations. celles-ci sont dues principalement à une photo ionisation à la longueur d'onde laser du niveau supérieur de la transition et à une destruction des miroirs par le flux VUV intense, ce qui réduit fortement l'énergie qu'il est possible d'extraire sous la forme d'un faisceau cohérent. Aussi, bien que plusieurs émisssions laser inteñses aient été mises en évidence dans les années 1970 ( $\mathrm{Ar}_{2}$ à $120 \mathrm{~nm}$, $\mathrm{Kr}_{2}{ }^{\star}$ à $132 \mathrm{~nm}, \mathrm{Xe}_{2}{ }^{*}$ à $\left.172 \mathrm{~nm}\right)^{8}$, il s'en est suivi un fort ralentissement des recherches sur ces systèmes dans les années 1980. Récemment un net regain d'intérêt est apparu pour ces systèmes comme lasers et, surtout, comme sources incohérentes à haut rendement.

Une étude des lasers $\mathrm{Xe}_{2}{ }^{*}$ et $\mathrm{Kr}_{2}{ }^{*}$ excités par un faisceau d'électrons dans des conditions d'impulsions longues a relativement faible densité de courant a montré que bien que le rendement de fluxorescence mesuré soit voisin de la limite théorique de $45-50 \%$, le rendement du laser $\mathrm{Xe}_{2}$ ne dépasse pas $1 \%$, par suite de la photo-absorbtion de l'excimère 
et le laser $\mathrm{K}_{2}{ }^{*}$ est au voisinage du seuil d'oscillation,9,10. Une autre étude, très récente, utilisant de l'argon à très haute pression (20-40 atm.), un faisceau d'électron à haute énergie et une cavité laser constituée d'un miroir en SiC et d'une fenêtre en $\mathrm{MgF}_{2}$ a permis d'extraire une énergje et une puissance laser égales respectivement à $80 \mathrm{~mJ}$ et fo $\mathrm{MW}$ en $5 \mathrm{~ns}$ à mihauteur 1 . La Fig.1 montre l'évolution $\mathrm{P}_{1}(\mathrm{t})$ de l'émission du laser $\mathrm{Ar}_{2}{ }^{*}$ en fonction de la pression pour une tension du canon constante. Enfin une étude theorique a montré la possibilité d'exciter efficacement les excimères Rg2 ${ }^{2}$ par décharge à haute pression ${ }^{12}$.
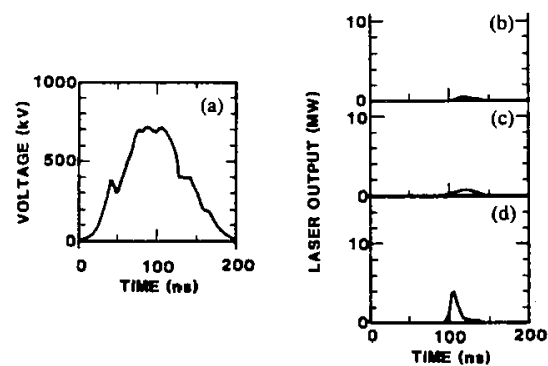

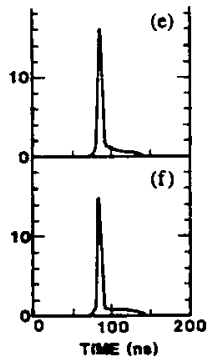

Fig. 1

Evolution dans le temps de la tension canon (a), et de l'émission laser de $\mathrm{Ar}_{2}$ à $126 \mathrm{~nm}$, (b) $P=22$ atm., (c) 23 atm.

(d) 25 atm., (e) 35 atm., (f) $40 \mathrm{~atm}$. (extrait de la Ref. 11)

Plusieurs études récentes ont montré expérimentalement que l'émission de fluorescence dans le VUV des excimères de gaz rares peut présenter des rendements importants (jusqu'à plusieurs dizaines de pour cent de l'énergie injectée dans le mjilieu) lorsqu'ils sont excités par des moyens aussi variés qu'une décharge mjicro-onde ${ }^{13,14}$, un rayonnement synchrotron ${ }^{15}$, une décharge à travers un diélectrique ${ }^{16,17}$ ou un faisceau de rayons $X$ durs ${ }^{18}$. Ces systèmes sont très attrayants comme source de photons VUV économiques et ont comme avantage supplémentaire de permettre un taux de répétition élevé. Ces systèmes font l'objet d'un autre article du même numéro du J. de physique ${ }^{19}$.

Plusieurs études d'excitation par décharge électrique de gaz rares en écoulement supersonique (détente adiabatique dans un jet et décharge à travers le col de la tuyère) ont été entreprises à la suite de l'annonce en 1989 de l'obtention d'une émission laser à 126 $\mathrm{NM}$ à partir de $\mathrm{Ar}_{2}{ }_{2} \mathrm{CO}^{\mathrm{O}}$. Les tentatives pour reproduire ces expériences ont jusqu'à présent été infructueuses les expériences effectuées dans, les autres laboratoires ayant permis d'obtenir jusqu'à très récemment des émissions jintenses $\left(\mathrm{Si}^{+}\right.$, etc..), mais pas d'émission

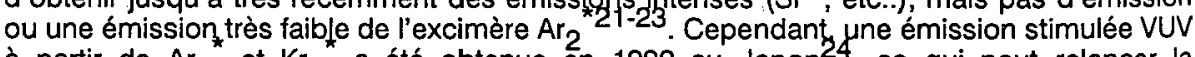
à partir de $\mathrm{Ar}_{2}$ et $\mathrm{Kr}_{2}$ a été obtenue ên 1992 au Japon ${ }^{24}$, ce qui peut relancer la controverse sur les possibilités d'obtenir un laser VUV à partir d'un jet supersonique de gaz rare excité par décharge. Les avantages d'un tel système seraient sa simplicité et un taux de répétition très élevé. La Fig. 2 représente le dispositif utilisé par T. Efthimiopoulos tandis que les Fig. 3 a et $3 \mathrm{~b}$ reproduisent respectivement les spectres d'émission VUV obtenus par $\mathrm{K}$. Kurosawa lors de l'excitation par décharge de Ar et $\mathrm{Kr}$ dans une détente supersonique (jet libre). Enfin, des émissions de fluorescence UV intenses des halogénures de gaz rares et de $\mathrm{XeO}$ viennent d'être obtenues au moyen de l'excitation par décharge, dans ung détente supersonique, de mélanges de gaz rares et de porteurs d'halogènes ou d'oxygène ${ }^{23}$.

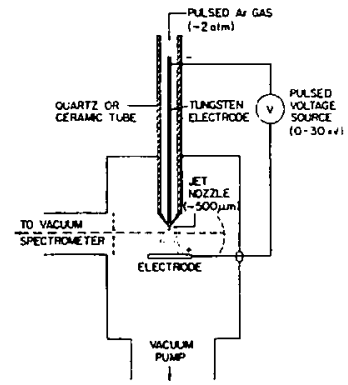

Fig. 2 Schéma du dispositif utilisé dans la Ref. 20
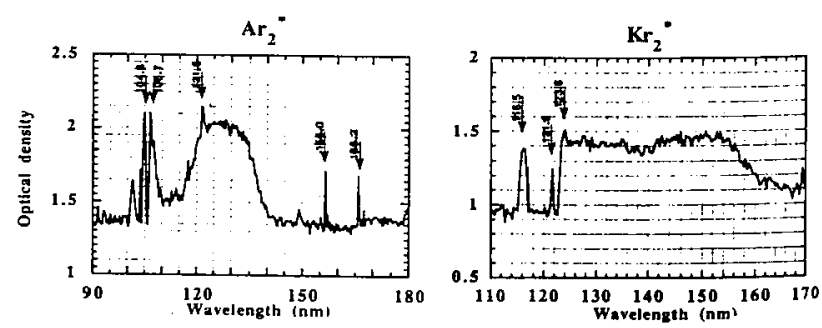

Fig. 3 Spectre d'émission VUV obtenu par décharge dans un jet supersonique, (a) argon, (b) Kripton (Ref. 24) 


\section{Excimères ioniques}

Une nouvelle classe de lasers à excimères basée sur les molécules d'excimères ioniques qui sont isoélectroniques aux excimères classiques a été proposée en 1985 indépendamment par R. Sauerbrey 25 , N.G. Basov ${ }^{26}$ et S. Iwata 27 et une première modélisation détaillée de la cinétique de mélanges de gaz rares et d'alcalins à haute pression et haute température a été effectuée ${ }^{28}$. Le tableau T. I représente les isoélectronicités entre les excimères neutres et les excimères ioniques. Depuis, plusieurs laboratoires ont étudiés théoriquement, numériquement et expérimentalement ces systèmes. Ces études ont porté essentiellement sur les systèmes alcalin-halogène $A^{+} X$ et gaz rare-alcalin $\mathrm{Rg}^{+} \mathrm{A}$ crées par l'excitation, à haute température, de mélanges gazeux soit par un faisceau de rayons $X$ mous créés par l'action d'un faisceau laser pulsé de grande puissance crête sur une cible, soit par un faisceau d'ions, soit par un faisceau pulsé d'électrons relativistes. L'excitation de ces systèmes, dont le niveau fondamental est dissociatif et le premier niveau excité relativement fortement lié, a permis d'obtenir un grand nombre d'émissions de fluorescence VUV-EUV. La durée de vie radiative de ces molécules ioniques est estimée à $1-100$ ns. Ces émissions sont souvent aussi intenses que les émissions des excimères $\mathrm{Rg}_{2}$ soumis à la même excitation à température ambiante.

\begin{tabular}{|c|c|c|c|c|}
\hline GROUPE & $\begin{array}{l}\text { EXCIMERE NEUTRE } \\
\text { (EXEMPLE) }\end{array}$ & $\begin{array}{l}\text { EXCIMERE IONIQUE } \\
\text { ISOELECTRONIQUE } \\
\text { (EXEMPLE) }\end{array}$ & ETAT FINAL & $\begin{array}{l}\text { MECANISME DE } \\
\text { PRODUCTION } \\
\text { DE L'EXCIMERE }\end{array}$ \\
\hline 1 & $\mathrm{Rg}^{\prime}+\mathrm{X}^{-}\left(\mathrm{KrF}^{*}\right)$ & $A^{++} X^{-}\left(\mathrm{AbF}^{+}\right)$ & $A+x$ & $\mathrm{He}^{+}+\mathrm{AX}$ \\
\hline II & $\mathrm{X}^{+} \mathrm{X}^{-}\left(\mathrm{Cl}_{2}\right)$ & $\begin{array}{c}\mathrm{Ag}^{++} \mathrm{Rg}\left(\mathrm{Ar}_{2}++\right) \\
\mathrm{Ag}^{++} \mathrm{X}^{-}\left(\mathrm{XeF}^{+}\right)\end{array}$ & $\begin{array}{l}\mathrm{Rg}^{+}+\mathrm{Rg}^{+} \\
\mathrm{Rg}^{+}+\mathrm{x}\end{array}$ & $\begin{array}{l}\mathrm{Rg}^{++}+2 \mathrm{Rg} \\
\mathrm{He}^{+}+\mathrm{RgX}\end{array}$ \\
\hline III & $\mathrm{Rg}_{2}{ }^{\star}\left(\mathrm{As}_{2}{ }^{*}\right)$ & $\mathrm{Rg}^{+} \mathrm{A}\left(\mathrm{ANNa}^{+}\right)$ & $\mathrm{RgA}^{+}$ & $\begin{array}{c}\mathrm{Rg}^{+}+\mathrm{Rg}+\mathrm{A} \\
\mathrm{Rg}_{2}^{+}+\mathrm{A}\end{array}$ \\
\hline
\end{tabular}

$T$. I isoélectronicités entre les excimères neutres et ioniques de gaz rare $(R g)$, d'alcalins $(A)$ et d'halogènes $(X)$

L'excitation des halogénures d'alcalin $\mathrm{CsF}, \mathrm{RbF}, \mathrm{KF}, \mathrm{CsCl}$ et $\mathrm{Nal}$ par un faisceau de rayons $X$ mous à permis l'observation d'émissions VUV large bande intenses des composés d'un alcalin lourg et d'un halogène léger $\mathrm{Cs}^{+} F(\lambda=185 \mathrm{~nm}), \mathrm{Rb}^{+} F(\lambda=130 \mathrm{~nm})$ et $\mathrm{Cs}^{+} \mathrm{Cl}$ $(\lambda=208 \mathrm{~nm})^{29-32}$. Il est à noter que les rayons $X$ mous excitent relativement facilement les couches internes électroniques des molécules $A^{+} X$ qui vont participer à l'état excimère. La Fig. 4 représente le diagramme d'énergie de la molécule CsF, tandis que la Fig. 5 montre le spectre de fluorescence VUV de la même molécule excitée par des rayons $X$ mous. Des études cinétiques et spectroscopiques détaillées ont été effectuées pour ces conditions. Les sections efficaces d'absorption et d'émission stimulée ont été calculées dans le cas de $\mathrm{Cs}^{+} \mathrm{F}\left(\sigma_{\text {stm }}=210^{-16} \mathrm{~cm}^{2} \text { et } \sigma_{\text {abs }}=2,710^{-18} \text { à } \lambda=185 \mathrm{~nm}\right)^{32}$. La population estimée de l'état superrieur est $10^{13}-10^{14} \mathrm{~cm}^{3}$ à $5 \mathrm{~mm}$ de la cible ${ }^{32}$. Des émissions VUV à partir du système $A^{+} X$ ont également été obtenues récemment lors de l'excitation de mélanges gaz rares- haloggénures d'alcalins excités à haute température et haute pression par un faisceau d'ions ${ }^{33,34}$ et par un faisceau pulsé d'électrons ${ }^{35-37}$. Des calculs Ab Initio des courbes de potentiel de ces molécules ont également été effectués ${ }^{38}$.

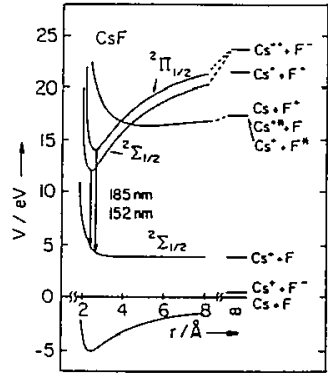

Fig. 4 énergie potentielle de CsF (Rer. 35)

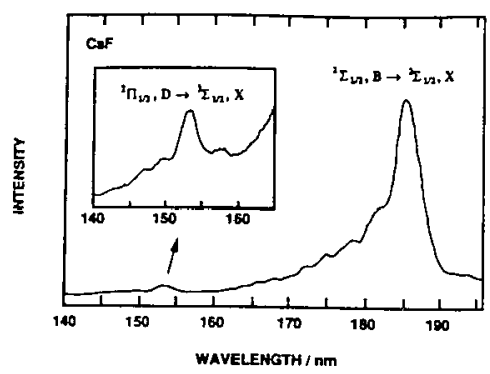

Fig. 5 Spectre de fluorescence VUV de CsF excité par X (Ref 32) 
L'excitation de divers mélanges de gaz rares et d'alcalins à haute température $(\mathrm{T}=700 \mathrm{~K})$ a permis d'observer un grand nombre d'émissions intenses d'excimères ioniques $\mathrm{Ar}^{+} \mathrm{A}$ depuis la première mise en évidence expérimentale de l'existence d'une telle émission en 1989 a partir de $\mathrm{Xe}^{+} \mathrm{Cs}(\lambda=161 \mathrm{~nm})$ et $\mathrm{Xe}^{+} \mathrm{Rb}(\lambda=164 \mathrm{~nm})$ au moyen d'un faisceau d'ion ${ }^{39-41}$, de rayons $X$ mous ${ }^{29}$, d'un faisceau d'électrons ${ }^{40-45}$ et très récemment d'une décharge à haute pression ${ }^{46}$. Ces études, entreprises souvent dans le but de réaliser un laser VUV accordable, n'ont pas encore abouti à une émission laser mais ont permis de progresser de façon importante dans la connaissance de la spectroscopie et de la cinétique de ces systèmes et notamment de montrer que l'état excimère est formé effjcacement par des réactions à trois corps entre les atomes de gaz rares et d'alcalin $40,47-49$. La Fig. 6 représente schématiquement le diagramme d'énergie de la molécule XeCs. Récemment a été mise en évidence une des émissions intense à partir de $\mathrm{Kr}^{+} \mathrm{Rb}(\lambda=134 \mathrm{~nm})$ lors $\mathrm{ge}$ l'excitation de mélanges $\mathrm{Ar} / \mathrm{Kr} / \mathrm{Rb}$ par un faisceau d'électrons en impulsions longues ${ }^{44,45}$ et en impulsions courtes ${ }^{42}$. De nombreuses émissions des excimères ioniques $\mathrm{Rg}^{+} \mathrm{A}$ entre 60 et $80 \mathrm{~nm}$ ont été obtenues très récemment par K. Petkau 40 au moyen d'une excitation par faisceau d'ions. Ces émissions proviennent des excimères ioniques $\mathrm{Ne}^{+} \mathrm{Li}, \mathrm{Ne}^{+} \mathrm{Na}$, $\mathrm{Ne}^{+} \mathrm{K}, \mathrm{Ne}^{+} \mathrm{Rb}, \mathrm{He}^{+} \mathrm{Li}, \mathrm{He}^{+} \mathrm{Na}$ et $\mathrm{He}^{+} \mathrm{K}$. Le tableau II représente les caractéristiques spectroscopiques de ces systèmes tandis que la Fig. 7 montre des spectres d'émission VUV de mélanges neon/alcalin à haute température excités par un faisceau d'ion. Des études de la cinétique des excimères ioniques $\mathrm{Rg}^{+} \mathrm{A}$ sont en cours dans plusieurs laboratoires, une des difficultés importantes étant la connaissance des constantes de vitesse des réactions clef à température élevée. Ces études ont déja permis de progresser notablement dans la connaissance de ces nouvelles molécules. Ainsi une constante de vitesse $\mathrm{k}=610^{-30} \mathrm{~cm}^{6}$ $\mathrm{s}^{-1}$ a été mesurée très récemment pour la réaction à trois corps ( $\mathrm{Ar}, \mathrm{Xe}, \mathrm{Rb}$ ) de création de l'excimère ionique $\mathrm{Xe}^{+} \mathrm{Rb}$ lors de l'excitation par un faisceau d'électrons de mélanges $\mathrm{Ar}$, $\mathrm{Xe}$ et $\mathrm{Rb}^{43}$. La section efficace d'émission stimulée de ces excimères ioniques est estimée à $10^{-17} \mathrm{~cm}^{2}$, valeur deux ordres de grandeur supérieure à la section efficace d'absorption. II n'a toutefois pas encore été possible d'obtenir d'émission laser à partir du système $\mathrm{Rg}^{+} \mathrm{A}$.

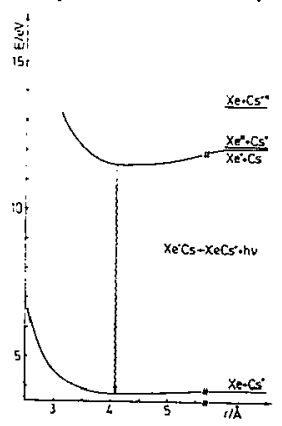

Fig. 6 énergie potentielle de $\mathrm{Xe}^{+} \mathrm{Cs}$ (Ref. 39)

\begin{tabular}{|c|c|c|c|c|c|c|c|c|}
\hline & $\stackrel{i}{i n n \mid}$ & $i$ & $\begin{array}{l}\mathrm{sv_{1 }} \\
\left\{\mathrm{cm}^{-1}\right\}\end{array}$ & $\begin{array}{l}3 v_{1} \\
\left(\mathrm{~cm} \cdot{ }^{\prime}\right)\end{array}$ & $\begin{array}{l}\Delta v_{1} \\
(\mathrm{~cm})\end{array}$ & $\left(\mathrm{s} \mathbf{v}_{1}\right.$ & $\begin{array}{l}D_{1} \\
(\mathrm{~cm} \cdot 1)\end{array}$ & $\begin{array}{c}D_{0} \\
(\mathrm{~cm} \cdot 1)\end{array}$ \\
\hline $\mathrm{Se}_{\mathrm{e}}=\mathrm{Li}$ & 80.70 & $\begin{array}{c}123920 \\
(123460)\end{array}$ & $\begin{array}{l}1040 \\
(830)\end{array}$ & $\begin{array}{c}340 \\
1310\}\end{array}$ & $\begin{array}{c}90 \\
(230)\end{array}$ & $\begin{array}{l}760 \\
\text { (570) }\end{array}$ & $\begin{array}{c}6800 \\
(4180)\end{array}$ & 970 \\
\hline $\mathrm{Ve}^{-} \mathrm{N}_{\mathbf{2}}$ & 79.40 & $\begin{array}{c}125940 \\
(123920)\end{array}$ & $\begin{array}{l}1060 \\
(830)\end{array}$ & $\begin{array}{c}290 \\
(270)\end{array}$ & $\begin{array}{c}100 \\
(200)\end{array}$ & $\begin{array}{l}600 \\
(470)\end{array}$ & $\begin{array}{c}6700 \\
\{3730)\end{array}$ & 570 \\
\hline $\mathrm{Ne}^{-} \mathrm{K}$ & 77.18 & $\begin{array}{c}129570 \\
(127550)\end{array}$ & 1190 & 150 & 70 & $\ldots$ & 4600 & 340 \\
\hline $\mathrm{Ne}_{\mathrm{e}} \cdot \mathbf{R b}$ & 76.66 & $\begin{array}{c}130450 \\
(128700)\end{array}$ & 1030 & 170 & $\cdots$ & $\cdots$ & 3700 & 270 \\
\hline $\mathrm{Ne}^{*} \mathrm{Cs}$ & $\ldots$ & $(130550)$ & $\cdots$ & $\ldots$ & $\cdots$ & $\ldots$ & .. & 200 \\
\hline Eток & \pm 0.05 & \pm 80 & \pm 30 & \pm 50 & \pm & \pm 90 & \pm 100 & \\
\hline
\end{tabular}

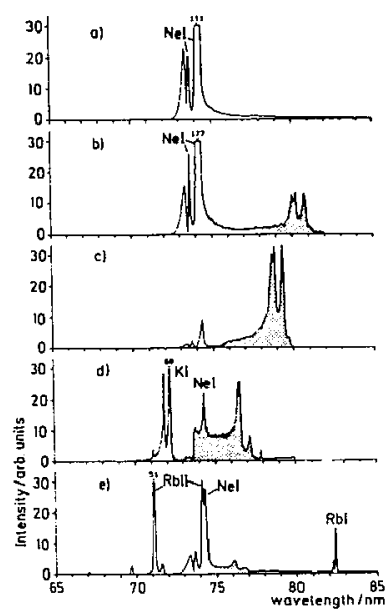

Ne pur

$\mathrm{Ne} / \mathrm{Li}$

$\mathrm{Ne} / \mathrm{Na}$

$\mathrm{Ne} / \mathrm{K}$

$\mathrm{Ne} / \mathrm{Rb}$

T. Il Caractéristiques spectroscopiques d'exciplexes ioniques $\mathrm{Rg}^{+} \mathrm{A}$ émettant dans l'EUV (Ref. 40)

Fig. 7 Spectre de fluorescence EUV d'excimères ioniques $\mathrm{Rg}^{+} \mathrm{A}$ (Ref. 40) 


\section{Décharges de surface sur ferrite formée}

La création de plasmas froids et denses produits à l'aide de décharges de surface ou de fils explosifs a fait l'objet ces dix dernières années d'un certain nombre d'études intensives ayant pour but le développement de nouvelles sources de préionisation ou de pompage direct, par rayonnement dans le proche ultraviolet, lultraviolet du vide ou l'extrême ultra violett, de lasers à gaz pulsés (exciplexes, laser à iode,.création de la molécule excitée $\mathrm{O}^{\star},(1 \Delta)$. Ceci a permis d'obtenir de nombreuses émissions laser de l'uV à l'infrarouge et en particulier une énergie laser de $98 \mathrm{~J}$ sur XeF $(C \rightarrow>A)$ à $490 \mathrm{~nm}(5 \mathrm{~J} /)^{50}$ et de $70 \mathrm{~J}$ sur $\mathrm{XeF}(\mathrm{B}-\mathrm{X} X)$ à $351 \mathrm{~nm} 50,51$ avec un rendement d'environ $1 \%$. On trouvera une excellente revue sur le ce type de lasers dans un ouvrage très récent de V.S. Zuev 52 .

Une nouvelle technique de création d'un plasma basée sur le principe d'une décharge électrique sur la surface d'une ferrite dite "formée" (création d'un filament amorphe $\left(\rho=10^{5} \mathrm{Ohms} / \mathrm{cm}\right.$ ) sur une des faces de la ferrite (structure initiale polycristalline, $\rho=10^{\circ}$ $\mathrm{Ohms} / \mathrm{cm})$ ), a été proposée en $1987^{53,54}$. Cette technique permet la création d'un plasma de surface émettant avec un rendement élevé dans un domaine spectral allant semble-t-il de l'ultra violet aux rayons $X,(\eta=20 \%$ dans la bande $140-200 \mathrm{~nm}$ et $45 \%$ dans la bande $140-$ $5000 \mathrm{~nm}$ ) et cela pendant une durée de l'ordre de 2 aे $5 \mu \mathrm{s}$. Cette nouvelle approche a l'avantage essentiel par rapport à la technique des fils explosifs de pouvoir fonctionner en régime répétitif ${ }^{53}$. La tension de seuil de formation du plasma est indépendante de la nature des gaz, de la pression et de la distance entre les électrodes placées à chaque extrémité du filament amorphe, ce qui n'est pas le cas des décharges de surface classiques (Téflon, $\left.\mathrm{Al}_{2} \mathrm{O}_{3}, \ldots.\right)$. Le mécanisme détaillé de cette décharge de surface sur ferrite n'est actuellement pas connu. Les Fig. 8 et 9 représentent respectivement le processus de formation du plasma émetteur dans une décharge sur ferrite formée et un spectre d'émission UV-VUV.

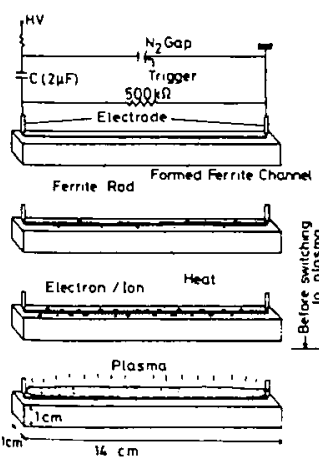

Fig. 8 Evolution du plasma dans une décharge sur ferrite formée (Ref. 54)

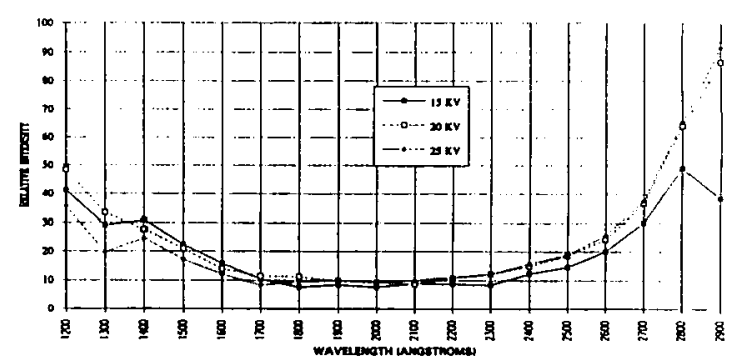

Fig. 9 Spectre de fluorescence UV-VUV type d'une décharge sur ferrite formée (Argon, 600 torrs) (extrait de la Ref. 58)

Actuellement trois principaux axes de recherches sont développés concernant les décharges de surfaces sur des ferrites formées : (a) l'étude des caractéristiques du plasma ${ }^{34-59}$, (b) les possibilités d'utilisation de cette source comme générateur d'électrons de préionisation ou comme générateur de faisceaux d'électrons pulsés relativistes ou de rayons $x^{60}$, (c) les possibilités d'utilisation de cette technique comme source de pompage par photolyse UV-VUV de lasers ${ }^{61,62}$. Un certain nombre de lasers ont ainsi été réalisés récemment par cette technique: laser $I_{2}\left(D^{\prime}-A^{\prime}\right) 61$, Laser $X e F(C \rightarrow A$ et $\left.B \rightarrow X)\right)^{62}$. On peut noter, en particulier, que l'étude fondamentale d'un plasma créé au moyen d'une décharge sur la surface d'une ferrite "formée" placée dans une cellule contenant différents gaz ( $\mathrm{Ar}, \mathrm{N}_{2}, \mathrm{CF}_{4}, \ldots$ ) pour une pression pouvant varier de quelques $10^{-6}$ torr à environ un bar est actuelement entreprise par plusieurs laboratoires dont le groupe Nouveaux Lasers de l'IMFM depuis $1990^{58,59}$. Ces recherches pourraient amener à la réalisation d'un laser à excimère répétitif de très longue impulsion $(5 \mu \mathrm{s})$ émettant dans le domaine spectral du bleu 
vert $(\mathrm{XeF}, \mathrm{C}-\mathrm{A}$ A) avec un rendement relativement élevé ainsi qu'une lampe flash accordable dans le VUV avec un rendement très élexés. Enfin, les recherches sur les possibilités des décharges de surface sur diélectrique ${ }^{52,63-66}$ et de plasmas créés par des

techniques de dynamique des plasmas ${ }^{67-70}$ pour l'excitation de lasers pulsés, sont actuellement également très actives. On peut noter, en particulier, que Yu. S. Protasov a obtenu très récemment une émission laser ayant une énergie de 3,5 joules à $\lambda=292 \mathrm{~nm}$ (laser $\mathrm{Br} 2$ ) par pompage au moyen d'un plasma thermique ayant une température de brillance dans l'UV-VUV $\mathrm{T}_{\mathrm{br}}=35000-40000 \mathrm{~K}^{69,70}$. Ce type de laser à pompage photolytique large bande pourrait être étendu vers de plus courtes longueurs d'onde en utilisant d'autres molécules d'halogène $(\lambda=157-342 \mathrm{~nm})$.

\section{Laser $\mathrm{KrCl}$ en régime d'impulsions longues}

Les lasers à exciplexes excités par décharge sont de plus en plus utilisés dans les domaines scientifiques et techniques. Cependant l'emploi des circuits d'excitation "classiques", tel le circuit C-L-C, limitent les caractéristiques de l'émission laser (courte durée d'impulsion et rendement relativement faible). Le développement récent du laser $\mathrm{XeCl}(\lambda=$ $308 \mathrm{~nm})$ à haut rendement $(\eta=3-4 \%)$ en régime d'impulsions longues (200 ns) a été permis par l'emploi d'une noyyelle méthode d'excitation, la double décharge à commutation magnétique rapide ${ }^{7-74}$. L'utilisation de ce type d'excitation entraîne, en plus de l'augmentation du rendement, une réduction des charges électrique, mécanique acoustique et optique sur les composants du laser ainsi qu'une amélioration de la qualité optique du faisceau par suite de l' augmentation d'un ordre de grandeur de la durée de l'émission laser. Enfin, l'augmentation de la durée d'impulsion permet une augmentation très notable de l'énergie laser UV qu'il est permis de transférer à distance au moyen de fibres optiques en silice $^{75}$, les phénomènes de destruction de la face d'entrée de la fibre, l'atténuation à deux photons et la formation de centres colorées limitant généralement la puissance optique UV instantanée qu'il est possible de transmettre à travers la fibre. Les applications de ce type de lasers sont nombreuses notamment dans les domaines de la médecine, du radar et des micro- techniques. Cependant les tentatives pour étendre ces lasers à impulsions longues vers de plus courtes longueurs d'onde n'ont pas jusqu'à très récemment donné de résultats satisfaisants, seul le laser $\operatorname{KrF}(\lambda=248 \mathrm{~nm})$ ayant permis d'obtenir des impulsions longues mais avec un rendement beaucoup plus faible $(<1 \%)$ que celui du laser $\mathrm{XeCl}$.

Au cours de l'étude des lasers à exciplexes de grande puissance moyenne à impulsions très longues et rendement élevé, une émission laser à $\lambda=222 \mathrm{~nm}$ (laser KrCl) en régime d'impulsions longues $(\Delta T=180 \mathrm{~ns}$ à mi-hauteur) a été mise en évidence pour la première fois en décembre 1991 à l'IMFM ${ }^{76}$, permettant ainsi d'augmenter d'un ordre de grandeur la durée d'impulsion de ce type de laser à très courte longueur d'onde. Au cours de ces expériences préliminaires mettant en oeuvre une préionisation par rayons $X$ et une excitation par double décharge à commutation magnétique, une énergie laser de $115 \mathrm{~mJ}$ a été extraite de mélanges $\mathrm{Ne} / \mathrm{Kr} / \mathrm{BCl}_{3}$ et $\mathrm{Ne} / \mathrm{Kr} / \mathrm{HCl}$ à $\mathrm{P}=3 \mathrm{Atm}$., à partir d'un faible volume actif $\left(V=25^{\star} 2,6^{\star} 1 \mathrm{~cm}^{3}\right)$, à travers un miroir de $20 \%$ de transmission. L'énergie et la durée de l'émission laser étaient sensiblement les mêmes en mode "commutateur magnétique" et en mode "charge résonnante". Le rendement par rapport à l'énergie stockée dans la ligne était de $0,85 \%$. Ce résultat nouveau peut avoir des retombées technologiques importantes, notamment pour les applications nécessitant le transfert d'énergies laser UV-VUV pulsées importantes par fibre optique. En effet, les problèmes d'interaction laser-fibre limitent actuellement fortement la puissance laser instantanée disponible pour l'utilisation des lasers à exciplexes à très courte longueur d'onde $(\lambda<308 \mathrm{~nm})$. La possibilité d'un rendement laser élevé $(\eta>1 \%)$, après optimisation de la cavité optique et du circuit de décharge, rend également très attrayant ce nouveau mode de fonctionnement du laser $\mathrm{KrCl}$.

\section{Autres sources VUV primaires}

Parmi les sources primaires VUV-EUV pour lesquelles des progrès notables sont apparus récemment, on peut citer les systèmes excités par rayons $X$ mous créés par l'action d'un faisceau laser pulsé de grande puissance crête sur une cible, ou un système optique 
original permet d'obtenir une relativement grande longueur de gain. Ce type d'excitation a permis d'obtenir la saturation de la transition laser pour de relativement faibles puissances de pompage dans les cas du laser Auger Xell à $109 \mathrm{~nm}$ et du laser $\mathrm{H}_{2}$ à $116 \mathrm{~nm}$ (bande de Werner) 79.79 . On peut citer également l'effet très favorable d'une excitation initiale de faible énergie sur l'excitation du laser Auger Xelll par faisceau d'X mous ${ }^{80}$. Le système NO excité optiquement à $\lambda=157 \mathrm{~nm}$ par un laser $F_{2}$ en présence d'un champ magnétique, qui a déjà

permis d'obtenir une émission cohérente à 218 et $226 \mathrm{~nm}^{81}$, peut permettre dans le futur d'obtenir plusieurs dizaines de raies laser entre 160 et $250 \mathrm{~nm}$. Des progrès importants ont également été obtenus sur leş lampes à halogénure de gaz rare en régime pulsé ou continf excitées soit par micro-ondes ${ }^{82,83}$ soit par une décharge pulsée à travers un diélectrique ${ }^{84}$, soit par une décharge continue ${ }^{85}$. Un rendement de fluorescence important à $\lambda=157 \mathrm{~nm}$ a' été prédit pour $\mathrm{F}_{2}$ excité par micro-ondes ${ }^{86}$. Enfin, des résultats notables ont été obtenus dans la miniaturisation des systèmes de production de faisceaux de rayons $X$ mous intenses par laser pulsé pour l'excitation de lasers EUV-VUV et le traitement des matériaux ${ }^{87-89}$.

\section{Conclusion}

La présente revue des progrès récents obtenus au plan international sur nouvelles sources photoniques VUV, sans prétendre à l'exhaustivité, montre des résultats importants dans ce domaine très prometteur en terme d'applications potentielles. Ces progrès concernent autant les sources laser que les sources incohérentes large bande de rendement élevé. En particulier, les sources VUV à excimères et les nouvelles sources à exciplexes ioniques ainsi que les sources incohérentes à très grande bande spectrale apparaissent très prométeuses. II reste cependant encore beaucoup de "physique" à faire dans ce domaine, notamment sur les sources à exciplexes laser ou fluorescentes.

\section{Références}

1. Voir par exemple les résumés de communications sur les sources VUV paru ces 5 dernières années dans les technical digests des conf. internationales CLEO et QELS (OSA). 2. Laser techniques in the extreme ultraviolet, AIP Conference Proceedings $N^{\circ} 119$, S.E. Harris et T.B. Lucatorto Ed., AIP (1984)

3. short-wavelengtht lasers and their applications, C. Yamanaka, Ed. Springer-Verlag (1988)

4. G. Eden, UV and VUV lasers: Prospects and applications, Optics News, 14 (1988)

5. Conference on future prospects and applications for UV and VUV lasers II, Santa barbara, mars 1990, Proceedings, Engineering Fondation, New York Ed. (1990)

6. M. Stehlé, UVX 92, à paraitre dans le Journal de Physique IV (1992).

7. P.W. Hoff, J.C. Swingle,C.K. Rhodes, Appl. Phys. Lett., 23, 245 (1973).

8. Excimer lasers, C.K. Rhodes Ed. 2d Ed., Springer-Verlag (1984).

9. D.J. Eckström et al., J. Appl. Phys. 64, 1679 (1988).

10. D.J. Eckström et al., J. Appl. Phys. 64, 1691 (1988).

11. K. Kurosawa et al., IEEE J. Quant. Electron. 27, 71 (1991).

12. K. S. Gochelashvili et al. LASERION'91, Munich, Juin 1991, Résumés, p 109.

13. K. Yoshizawa, M. Taki, K. Tachibana, S. Moriyama, Appl. Phys. Lett. 59 , 1678 (1991)

14. O.A. Zakharova et al., Sov. J. Quantum Electron. 20, 813 (1990)

15. E. Morikawa et al., J. Chem. Phys. 91, 1469 (1989).

16. B. Gellert, U. Kogelschatz, Appl. Phys. B, 52, 14 (1991)

17. K. Stockwald, V. Schorpp, M. Neiger, C. Beneking, ICPIG, Pisa, Juillet 1991, P. 95 (1991)

18. C. Cachoncinlle et al., J. Phys. D: Appl. Phys. 23, 984 (1990)

19. J.M. Pouvesle, D. Pigache, UVX 92, à paraitre dans le Journal de Physique IV (1992).

20. T. Efthimiopoulos, B.P. Stoicheff, R.I. Thompson, Opt. Lett. 14, 624 (1989).

21. F. Kannari, F. Sato, M. Obara, 8th GCL, Madrid sept. 1990, SPIE Vol. 1397, 493 (1991)

22. H. Phillips et al., IEEE J. Quant. Electron., 27, 95 (1991)

23. R.B. Jones, J.H. Schloss, J.G. Eden, J. Appl. Phys. 71, 1674 (1992)

24. W. Sasaki, K. Kurosawa, S. Matsusono, Communication CTuH5, technical digests, p.112, conférence internationale CLEO (OSA), Anaheim, Californie, Mai 1992.

25. R. Sauerbrey, H. Langhoff', IEEE J. Quantum Elecron., QE21, 179 (1985)

26. N.G. Basov et al., Sov. J. Quant. Electron., 15, 1455 et 1461 (1985) 
27. S. Iwata, N. Sato, J. Chem. Phys., 82, 2346 (1985)

28. N.G. Basov et al., Sov. J. Quantum Electron., 17, 106 (1987).

29. S. Kubodera, L. Frey, P.J. Wisoff, R. Sauerbrey, Opt. Lett., 13, 446 (1989).

30. L. Frey, S. Kubodera, P.J. Wisoff, R. Sauerbrey, J. Opt. Soc. Am. B 6, 1529 (1989).

31. T.T. Yang, V.T. Gylys, D.G. Harris, J. Opt. Soc. Am. B 6, 1536 (1989).

32. S. Kubodera, P.J. Wisoff, R. Sauerbrey, J. Opt. Soc. Am. B 9, 10 (1992).

33. F. Steigerwald et al., Opt. Comm. 56, 240 (1985).

34. F. Steigerwald, F. Eimmert, H. Langhoff, W. Hammer, J. Chem Phys. 88, 7376 (1988).

35. Da Xing, K.I. Ueda, H. Takuma, Jap. J. Appl. Phys, 30, L1012 (1991).

36. V.T. Gylys et al., Laser '89, New Orleans, déc. 1989, Proc. p. 153, STS Press Ed. (1990)

37. M. Shumann, , H. Langhoff, J. Chem. Phys., 91, 5440 (1989)

38. D. Xing, K. Ueda, H. Takuma, Chem. Phys. Lett., 163, 193 (1989)

39. J. Fiedler et al., Z. Phys. D11, 141 (1989)

40. K. Petkau et al., J. Chem. Phys. 94,7769 (1991).

41. M. Mantel, G. Herre, H. Langhoff, K. Petkau, W. Hammer, J. Phys. B 23, 4111 (1990).

40. P.S. Millar et al., Appl. Phys. Lett., 55, 2176 (1989)

41. M. Shumann, H. Langhoff, J. Chem. Phys. 91, 5440 (1989).

42. D. Xing, K. I. Ueda, H. Takuma, Appl. Phys. Lett., 59, 1028 (1991)

43. H.M.J. Bastiaens et al., Appl. Phys. Lett., à paraitre (1992).

44. Th. Chauvet, Thèse, Université d'Aix-Marseille II (1991).

45. Ph. Delaporte et al., Laser ' 91 , Anaheim, déc. 1991, Proc. à paraitre, STS Press (1992).

46. D. Lo, J.L. Lawness, Opt. Comm. 86, 151 (1991)

47. M. Mantel et al., à paraitre (1992).

48. S. Iwata, S. Nanbu, H. Kitajima, J. Chem Phys. 94,3707 (1991)

49. D. Xing, K. I. Ueda, H. Takuma, Jap. J. Appl. Phys, 30, 3533 (1991).

50. V.S.Zuev et al., Sov. J. Quant. Electron. 16, 1665 (1986).

51. V.S.Zuev et al., Sov. J. Quant. Electon. 19, 748 (1989).

52. V.S. Zuev, L.D. Mikheev, Photochemical lasers, Harwood Academic, Publ. (1991)

53. K. Watanabe et al., 7th GCL, Vienne, Aout 1988, SPIE, Vol 1031, 450 (1989)

54. K. Watanabe, S. Kashiwabara, R. Fujimoto, Appl. Phys. Lett. 50,629 (1987)

55. S. Kashiwabara, K. Watanabe, R. Fujimoto, J.Appl. Phys. 62, 787 (1987)

56. S. Kashiwabara, K. Watanabe, R. Fujimoto, J.Appl. Phys. $\underline{63}, 2514$ (1988)

57. X.F. Han, T. Sakai, G.J. Hirst, R. Sauerbrey, F.K. Tittel, Phys. Lett. A 137, 34, (1989).

58. R.C. Sze, M. Sentis, Laser '91, Anaheim, déc. 1991, Proc. à paraitre, STS Press (1992).

59. M. Sentis Ph. Granier, F. Chazaud, W.I. Marine, M. Gerry, R.C. Sze, EMRS 1992 Spring Meeting, ICEM 92, Strasbourg, Juin 1992, Proceedings à paraitre (1992).

60. K. Watanabe, K. Furusho, S. Kashiwabara, R. Fujimoto, J. Appl. Phys, 68, 5059 (1990).

61. S. V. Mit'ko et al., Soviet Physics, Lebedev Inst. reports, 2, 59 (1989).

62. R.W.F. Gross, L.E. Schneider, S.T. Amimoto, Appl. Phys. Lett. 53, 2365 (1988).

63. R.E. Beverly III, J. Appl. Phys., 60,104 (1986).

64. R.E. Berverly III, 8th GCL, Madrid, Septembre 1990, SPIE, Vol 1397, 581 (1991).

65. R.E. Beverly III, Appl. Phys. Lett., 58, 1024 (1991).

66. R.E. Beverly III, J. Appl. Phys., 69, 3830 (1991).

67. A.S. Kamrukov et al., 8th GCL, Madrid, Septembre 1990, SPIE, Vol 1397, 137 (1991).

68. R.T. Abashevet al., Sov. J. Quant. Electron., 21, 498 (1991).

69. V.V. Boev et al., Sov. J. Quantum Electron. 21, 1329 (1991)

70. A.S. Kamrukov, N.P. Kozlov, Yu. S. Protasov, Comm. CTuH6, technical digests, p 114, CLEO'92 (OSA), Anaheim, Californie, Mai 1992.

71. C.H. Fisher et al., Appl. Phys. Lett. 48, 1574 (1986)

72. R.S. Taylor, K.E. Leopold, J. App. Phys., 65, 22 (1989)

73. J.W. Gerritsen, A.L. Keet, G.J. Ernst, W.J. Witteman, J.Appl. Phys., 67, 3517 (1989)

74. J.M. Hueber et al. Opt. Commun., 85, 237 (1991)

75. R.K. Brimacombe, R.S. Taylor, K.E. Leopold, J. Appl. Phys. 66, 4035 (1989)

76. J.M. Hueber, B.L. Fontaine, N. Bernard, B.M. Forestier, M.L. Sentis,Ph. C. Delaporte, Tech. note IMFM-NL 1992-1 (1992); soumis pour publication dans Appl. Phys. Lett.

77. M.H. Sher, S.J. Benerofe, J.F. Young, S.E. Harris, J. Opt. Soc. Am. B 8, 114 (1991).

78. S.J. Benerofe et al., Phys. Rev. Lett. 66, 3136,(1991).

79. T.E. Sharp, M.J. Byrd, J.F. Young, Comm. CTuH1, technical digests, p 110, conférence internationale CLEO (OSA), Anaheim, Californie, Mai 1992.

80. M.H. Sher, S.J. Benerofe, J. Opt. Soc. Am. B 8, 2437 (1991). 
81. S.M. Hooker, A.M. Haxell, C.E. Webb, Appl. Phys., B 54, 120 (1992).

82. I. Nakamura, F. Kannari, M. Obara, Appl. Phys. Lett., 57, 2057 (1990).

83. S.B. Hassal, E. A. Ballik, J. Appl. Phys., 70, 1042 (1991).

84. D. Strobl et al., ICPIG, Pisa, Juillet 1991, Proceedings p. 956 (1991).

85. R.S. Taylor, K.E. Leopold, K.O. Tan, Appl. Phys. Lett. 59, 525 (1991).

86. T. Hatakeyama, F. Kannari, M. Obara, Appl. Phys. lett., 59, 387 (1991).

87. C.H. Skinner, D. Kim, L. Polanski, W. Tighe, K. Krushelnik, S. Suckewer, QELS'92 (OSA), Anaheim, Californie, comm. QThG3, technical digests, p 252, Mai 1992.

88. H. Sekiguchi, H. Hara, N. Tadokoro, H. Tajima, T. Mochizuki, , CLEO'92 (OSA), Anaheim, Californie, comm. CTuE3, technical digests, p 92, Mai 1992.

89. J.N. Broughton, R. Fedosejevs, Appl. Phys. Lett., 60, 1818 (1992). 